\title{
Ocular suitability of Fertilizer (Urea) doped Semiorganic NLO single Crystal: Tri-Glycine potassium chloride
}

\author{
S. Thiyagaraj \\ Research Scholar \\ Karpagam University \\ Coimbatore, INDIA
}

\author{
G. Meenakshi \\ Phd, Associate professor \\ K.M Centre for PG Studies, \\ Puducherry, INDIA
}

\begin{abstract}
Urea of different percentage in molecular ratios was added to synthesized Tri-Glycine Potassium Chloride (TGPC) and assent to grow for some months to study the behavioral changes of Tri-glycine potassium chloride by the effect of Urea. The grown crystal was first characterized by UV [1] studies and it was analyzed that the transparency was reduced by a blow of fertilizer and a small change occurred in its wavelength and the absorbance range. This research is trying to be authenticating the causes by an impact of Urea on TGPC through the SEM and FTIR studies.
\end{abstract}

\section{Keywords}

Semiorganic, Nonlinear optical, Slow Evaporation, UV, SEM and FTIR.

\section{INTRODUCTION}

In modern year organic - inorganic mixture materials have attracted huge consideration. In particular, the present fascinating field of research is to synthesize, grow and characterize semi organic NLO crystals. There are so many attempt has been made to synthesize and grow number of NLO semi organic crystals, the discussion from that shows semi organic crystal posses both the good qualities of host organic materials and additive-inorganic material [2].The research with Single crystals of urea thiourea with mercuric chloride, sulphate, semi-organic nonlinear optical materials, having good second harmonic generation. Its UV-Vis spectra showed that the crystals had a wide optical window, no absorbance and good optical transmittance in the entire visible region. The FTIR analysis confirms the presence of functional groups in the grown crystals [3].The research related with amino acid single crystal like glycine with zinc chloride having good application in the field of NLO. [4]

A Single crystal of L-Glycine was grown from aqueous solution by slow evaporation method. The optical properties of the grown crystals were studied using UV-Vis spectral analysis. Optical absorption study reveals the transparency of the crystal in the entire visible region. This shows it has good transparency and the powder X-ray diffraction analysis confirms the crystalline nature [5]. Similarly, the Effect of amino acid (proline) doped on triglycine sulphate has good dielectric properties and also its Curie temperature increases when we doped with inorganic materials [6]. Good, and optical high qualities single crystal of GLO has been grown by slow evaporation techniques, its XRD result confirm that it is a monoclinic crystal and the optical absorption studies infer that the crystal possess very low absorption in the entire visible and IR region. Due to the presence of high transference range, high damage threshold values and high second harmonic efficiency crystal may be used for NLO application [7]. A research from pure triglycine sulphate (TGS) and LiSO4-doped TGS crystals were investigated with the help of slow evaporation method. It has good NLO property and electrical conductivity property. It has a good dielectric, microhardness, and thermal characters. And this crystal was found highly transparent and full faced. The direct current conductivity is found to increase with temperature as well as dopants concentrations. Curie temperature remains the same for pure and doped crystals, but dielectric constant and dielectric loss increases with dopants concentration. The experimental result shows the suitability of the grown crystal for optoelectronic applications [8].

Above form the literature review implies that, the commercial use of glycine is, for nuclear magnetic resonance (NMR) spectroscopists, glycine can serve as a standard for checking and calibrating spectrometer performance or utilizing the various isotopic enrichments commercially available for quick implementing and demonstrating a variety of techniques like rotational resonance. In general, the doped tri-glycine potassium chloride has some greater advantage when compared with single TGPC because it overcomes the disadvantage of single TGPC. Such as, ferroelectric domains possess high mobility at room temperature, depolarization by electrical and thermal means and microbial contamination with time during the growth and low Curie point and so on .These are done by adding Suitable impurities to TGPC crystals .This research is trying to enrich the changes in general properties of TGPC by adding Urea fertilizer of different molecular ratios and examined the changes through SEM and FTIR studies. In this investigation the urea acts as an impurity and it influences some changes in the general properties on tri-glycine potassium chloride. Generally triglycine potassium chloride finds some applications in the fabrication of capacitors, transducers and sensors.

\section{EXPERIMENTAL}

\subsection{Synthesis and crystal growth}

The Glycine (AR Grade) and Potassium Chloride (AR Grade) were re-crystallized for the needs of purity and good result. After that, the titled Single Crystal was synthesized by taking a molecular ratio of 3 percent of Glycine (Merck) added with $1 \%$ of Potassium Chloride (Merck) and it was dissolved in triple distilled water. The $\mathrm{pH}$ value of the super-saturated solution is kept at 6.0. The solution was filtered and transferred to a Petri dish for the seed preparation [7]. The fertilizer component named urea is added by considerable molecular ratio $(3: 1: 1)(0.62589 \mathrm{~g}: 0.20718 \mathrm{~g}: 0.1669 \mathrm{~g})$ with TGPC and once again it is dissolved with triple distilled water and transferred into a Petri dish for crystallization. The sample of seeds is suspended in the $500 \mathrm{ml}$ beaker that contains same supersaturated solution and placed lonely for some days for 
the growth process. The harvested grown crystal was taken and grained finely for Character studies.

\section{CHARACTERIZATION}

The grown crystals were harvested and grained finely for studying its characterizations. The optical activity of urea doped tri-glycine potassium chloride was investigated by UV-Vis spectrometer and its functional groups analysis were taken by FT-IR investigation and also the grain size of titled crystal was taken by SEM studies.

\section{RESULT AND DISCUSSION}

\subsection{UV-Vis Studies}

The optical activity of titled crystal was studied by UV-Vis spectrometer. UV absorption, Reflection and transmission studies were carried out by the spectrometer. The comparison graphs were plotted for each recorded value by the use of Orgin 6 software [1]. The investigation result implies that, the transmission wave length appears form $195 \mathrm{~nm}$ to $200 \mathrm{~nm}$ and absorption wave length lies at zero line. This is the basic qualification of NLO materials and therefore it may be used for optical applications. The comparison graphs[ Fig-1,2 ] of UV absorption, Reflection, transmission shows that, there is a change in intensity by doping Tri-glycine potassium chloride with Urea but there is no modulations in the wave lengths.

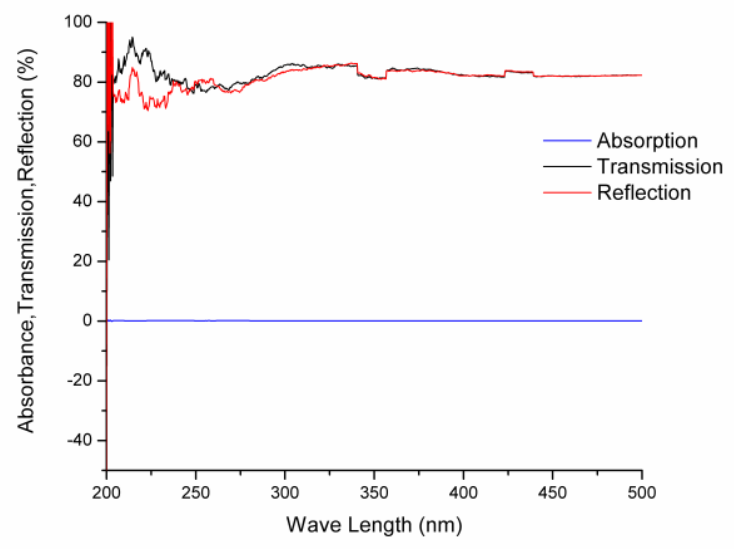

Fig. 1. UV-Vis graph of Tri-Glycine Potassium chloride without Urea doped

\subsection{FTIR studies:}

The grown crystals were subjected to FT-IR analysis. The sample prepared with $\mathrm{KBr}$ in the form of pellets. The FT-IR spectrum of tri-glycine potassium chloride was recorded in the region $4000-400 \mathrm{~cm}^{-1}$ employing Shimadzu IFS 66V spectrometer [Fig-3, 4].

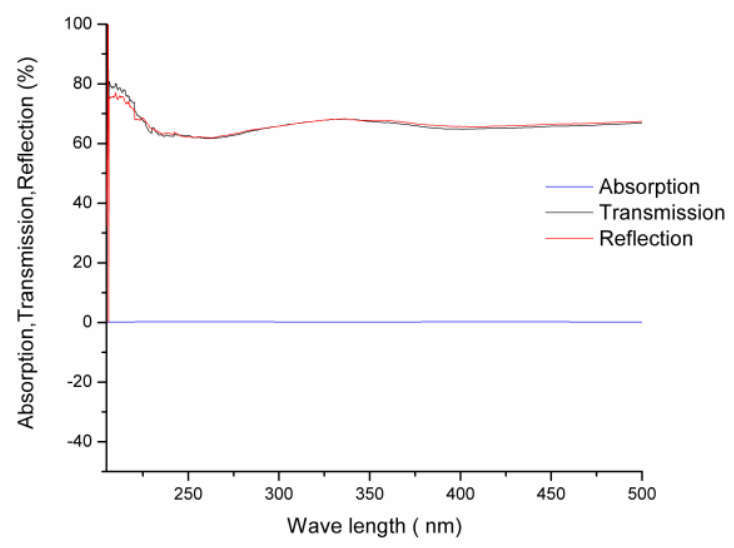

Fig.2. UV-Vis graph of Tri-Glycine Potassium chloride doped with Urea

\subsubsection{FTIR studies for TGPC without Urea doped}

\subsubsection{O-H Bonding}

Carboxylic acids can bond intermolecularly with solvent that are proton accepter, then the FT-IR spectra falls in such solvent band near $3100 \mathrm{~cm}^{-1}$. This is due to $\mathrm{O}-\mathrm{H}$ bonding. Similarly, in the FT-IR spectral line of TGPC single crystal, a line appears at $3105 \mathrm{~cm}^{-1}$. Therefore, a carboxylic acid in amino acid group intermolecularly bond with proton accepter solvent, which is potassium chloride.

\subsubsection{2. $\mathrm{NH}^{+}$Stretching Vibrations:}

The broad envelope in the high energy region between $3100 \mathrm{~cm}^{-1}$ and $2600 \mathrm{~cm}^{-1}$ occurs in FT-IT spectrum indicates that, there is $\mathrm{NH}_{3}{ }^{+}$stretching vibrations presented in the sample and also the region of absorption bands extends to about $2000 \mathrm{~cm}^{-1}$ due to multiple combination and overtone bands. The FT-IR spectrum of Tri-Glycine potassium chloride sample having spectral strong band between $3105 \mathrm{~cm}^{-1}$ and $2608 \mathrm{~cm}^{-1}$. Hence, these lines prove that $\mathrm{NH}_{3}^{+}$stretching vibrations are presented in the sample. And this region also extent up to 2169 this is due to the prominent band near $2000 \mathrm{~cm}^{-1}$ and $2200 \mathrm{~cm}^{-1}$ may be assigned to a combination of the asymmetrical $\mathrm{NH}_{3}{ }^{+}$bending vibrations and torsional oscillation of the $\mathrm{NH}_{3}{ }^{+}$group. The $\mathrm{NH}_{3}{ }^{+}$stretching region shows broad bands characteristics of hydrogen bonding ${ }^{[11]}$.

\subsubsection{Torsional Motion of $\mathrm{NH}_{3}^{+}$:}

In amino acid group the torsional motion of $\mathrm{NH}_{3}^{+}$groups appears near $500 \mathrm{~cm}^{-1}$. This is due to N-H stretching vibration. Here, the spectrum of TGPC having a normal spectral line exactly at the wave number of $505 \mathrm{~cm}^{-1}$. Therefore, the specimen TGPC having $\mathrm{NH}_{3}^{+}$torsional motions. This is due to $\mathrm{NH}_{3}^{+}$stretching vibration.

\subsubsection{C-N stretching vibration:}

Weak to medium intensity bands for un conjugated $\mathrm{C}-\mathrm{N}$ bond in primary, secondary and tertiary aliphatic amines occurs in the region of $1000 \mathrm{~cm}^{-1}$ to $1250 \mathrm{~cm}^{-1}$. They are due to coupled $\mathrm{C}-\mathrm{N}$ stretching mode. This depend the nature of the amine and substitution on the alpha carbon atom. Therefore the spectral line for $\mathrm{C}-\mathrm{N}$ stretching vibration occurs at the wave number of 
$1075 \mathrm{~cm}^{-1}$. Similarly, The TGPC having a Normal peak wave number at $1043 \mathrm{~cm}^{-1}$.

\subsubsection{5. $\mathrm{NH}_{2}$ Wagging:}

Theoretically $\mathrm{NH}_{2}$ wagging occurs at a broad absorption at approximately $800 \mathrm{~cm}^{-1}$. Here the small broad band occurs near $890 \mathrm{~cm}^{-1}$. This is due to $\mathrm{NH}_{2}$ stretching vibrations. The above discussions, observed, calculated FTIR frequencies and its assignments are given in table 1.

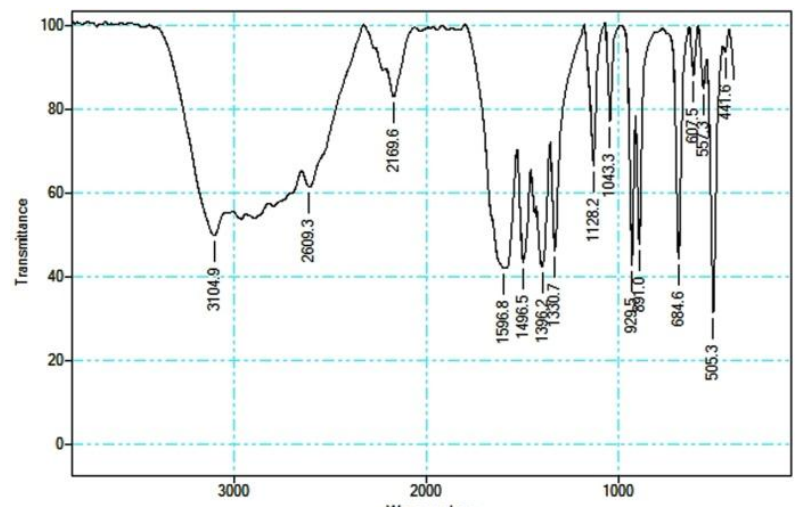

Fig. 3. FTIR spectrum of Tri-Glycine potassium Chloride without Urea doped

Table: 1. Observed ${ }^{[9]}$ and calculated FTIR frequencies $\left(\mathrm{cm}^{-1}\right)$ and assignments for Tri-glycine potassium chloride without Urea doped.

\begin{tabular}{|c|c|c|}
\hline $\begin{array}{c}\text { Obs. frequency } \\
\left(\mathbf{c m}^{-1}\right)\end{array}$ & $\begin{array}{c}\text { Cal. frequency } \\
\left(\mathbf{c m}^{-1}\right)\end{array}$ & $\begin{array}{c}\text { Assignment of } \\
\text { vibration }\end{array}$ \\
\hline 3200 & $3105(\mathrm{~W})$ & $\mathrm{O}-\mathrm{H}-$ Stretching \\
\hline $3100-2600$ & $3105-2608(\mathrm{~W})$ & $\mathrm{NH}^{+}$- Stretching \\
\hline $2200-2000$ & $2169(\mathrm{~S})$ & $\begin{array}{c}\mathrm{NH}^{+}{ }_{3} \text { asymmetric } \\
\text { bending and torsional }\end{array}$ \\
\hline $1550-1480$ & $1583-1495(\mathrm{~W})$ & $\begin{array}{c}\mathrm{NH}^{+} \text {Symmetric } \\
\text { bending }\end{array}$ \\
\hline $1300-1500$ & $\begin{array}{c}1495,1396,1330, \\
(\mathrm{~W})\end{array}$ & $\begin{array}{c}\text { Carboxylate anion } \\
\text { stretching }\end{array}$ \\
\hline 1075 & $1043(\mathrm{~N})$ & $\mathrm{C}-\mathrm{N}$ stretching \\
\hline Above 800 & $890(\mathrm{~W})$ & $\mathrm{NH}{ }_{2}$ wagging \\
\hline $800-650$ & $890-685(\mathrm{~W})$ & $\mathrm{N}-\mathrm{H}$ bending \\
\hline 775 & $685(\mathrm{~W})$ & $\mathrm{C}-\mathrm{Cl}$ stretching \\
\hline 500 & $505(\mathrm{~W})$ & Torsional mode \\
\hline
\end{tabular}

*W-weak: S-strong: N-normal

\subsubsection{FTIR study of TGPC doped with Urea}

\subsubsection{Hydrogen stretching vibration}

Carboxylic acids can bond intermolecularly with solvent that are proton accepter, then the FT-IR spectra measured in such solvent band falls near $3600 \mathrm{~cm}^{-1}$. This is due to Hydrogen bonding. Similarly, in the FT-IR spectral line of Urea doped TGPC single crystal, a line appears at $3606 \mathrm{~cm}^{-1}$. Therefore, a carboxylic acid in amino acid group intermolecularly bond with proton accepter solvent, which is potassium chloride.

\subsubsection{2. $N$-H Stretching vibration}

The spectrum of urea complex interprets very similar to those of the amino complexes. If bonding of urea to a metal occurs through oxygen, this may result in decrease of the $\mathrm{C}=\mathrm{O}$ stretching frequency due to higher contribution, but no appreciable change in the $\mathrm{N}-\mathrm{H}$ stretching mode. This may be due to the fact that in free urea, the N-H bond is involved in intermolecular hydrogen bonding in the solid state. $\mathrm{C}-\mathrm{N}$ stretching frequency increases considerably. In general $\mathrm{N}-\mathrm{H}$ stretching occurs at $3400 \mathrm{~cm}^{-1}$ to $3600 \mathrm{~cm}^{-1}$. Here, the spectral lines of urea doped TGPC appears at $3417 \mathrm{~cm}^{-1}$ is of very high intensity.

\subsubsection{C-C Ring stretching vibration}

Generally the skeletal vibration involving in C-C ring Stretching observed in the range of $1400 \mathrm{~cm}^{-1}$ to $1500 \mathrm{~cm}^{-1}$. When we doping urea with tri-glycine potassium chloride, there are two peaks appears very near to each other in the region of $1405 \mathrm{~cm}^{-1}$ and $1494 \mathrm{~cm}^{-1}$. This confirms that there is a strong C-C ring stretching invoked in these ratios.

\subsubsection{C-H out of plane bending vibration}

The $\mathrm{C}-\mathrm{H}$ out of plane bending vibration of Tri-glycine potassium chloride occurs due to the isolated hydrogen atoms existing in that sample. Generally the spectrum occurs in the region between $650 \mathrm{~cm}^{-1}$ and $1000 \mathrm{~cm}^{-1}$. This FTIR spectrum of TGPC having two spectral line in this region such as $687 \mathrm{~cm}^{-1}$ and $859 \mathrm{~cm}^{-1}$. This shows that it has $\mathrm{C}-\mathrm{H}$ out of plane bending vibration one has near $1000 \mathrm{~cm}^{-1}$ having high intensity and other having medium intensity which is near to $650 \mathrm{~cm}^{-1}$ region.

\subsubsection{5. $\mathrm{N}$ - $\mathrm{H}$ bending vibration}

The N-H bending band of medium intensity occurs very near to $650 \mathrm{~cm}^{-1}$ to $800 \mathrm{~cm}^{-1}$. The urea doped Triglycine potassium chloride spectrum occurs at $687 \mathrm{~cm}^{-1}$. This is because of N-H bending vibration and also the impact of urea on TGPC.

\subsubsection{6. $\mathrm{C}$-O stretching vibration}

If the compounds contain polar groups then the peaks appear very near in the region at $1000 \mathrm{~cm}^{-1}$ to $1300 \mathrm{~cm}^{-1}$. TGPC doped with urea has the spectral line at $1025 \mathrm{~cm}^{-1}$. It shows that there is a $\mathrm{C}-\mathrm{O}$ stretching vibration presented in this sample. The above discussions, observed, calculated FTIR frequencies and its assignments are given in table 2 .

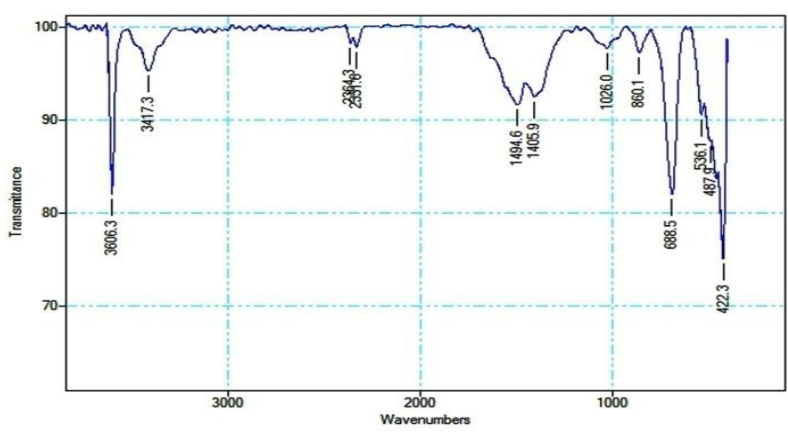

Fig.4. FTIR spectrum of Tri-Glycine potassium Chloride doped with Urea 
Table: 2. Observed and calculated FTIR frequencies $\left(\mathrm{cm}^{-1}\right)$ and assignments for Tri-glycine potassium chloride doped with urea.

\begin{tabular}{|c|c|c|}
\hline $\begin{array}{c}\text { Observed } \\
\text { frequency } \\
\left(\mathbf{c m}^{-\mathbf{1}}\right)\end{array}$ & $\begin{array}{c}\text { Calculated } \\
\text { frequency }\left(\mathbf{c m}^{-\mathbf{1}}\right)\end{array}$ & $\begin{array}{c}\text { Assignment of } \\
\text { vibration }\end{array}$ \\
\hline 3600 & $3606(\mathrm{~W})$ & Hydrogen bonding \\
\hline $3400-3600$ & $3417(\mathrm{~S})$ & N-H Stretching \\
\hline $1494-2331$ & $1494-2331(\mathrm{~S})$ & Can not identify \\
\hline $1400-1500$ & $1405-1494(\mathrm{~N})$ & C-C Stretching \\
\hline $1000-1300$ & $1025(\mathrm{~S})$ & C-O stretching \\
\hline $1000-650$ & $859(\mathrm{~S})$ & $\begin{array}{c}\text { C-H out of plane } \\
\text { bending }\end{array}$ \\
\hline $1000-650$ & $687(\mathrm{~N})$ & $\begin{array}{c}\text { C-H out of plane } \\
\text { bending }\end{array}$ \\
\hline $800-650$ & $687(\mathrm{~N})$ & N-H bending \\
\hline 775 & $536-400(\mathrm{~N})$ & Can not Identify \\
\hline
\end{tabular}

*W-weak: S-strong: N-normal

\subsection{SEM Analysis}

The SEM images for urea doped and without doped triglycine potassium chloride was investigated by scanning electron microscope. For undoped urea with potassium chloride the grain size appears between 2 micro meters and 5 micro meters [Fig.5], but when it is doped with Urea the grain size may start to reduce nano meter range. This is visible in the SEM photographs [Fig.6].
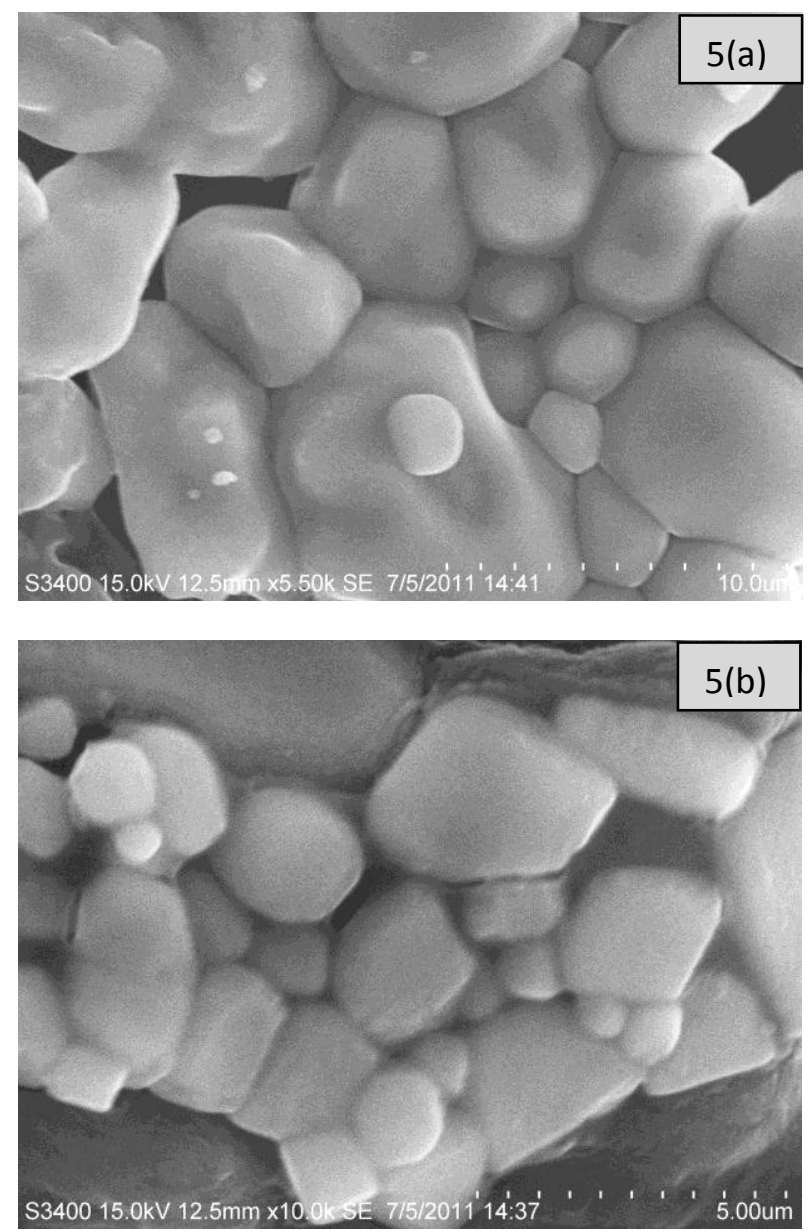
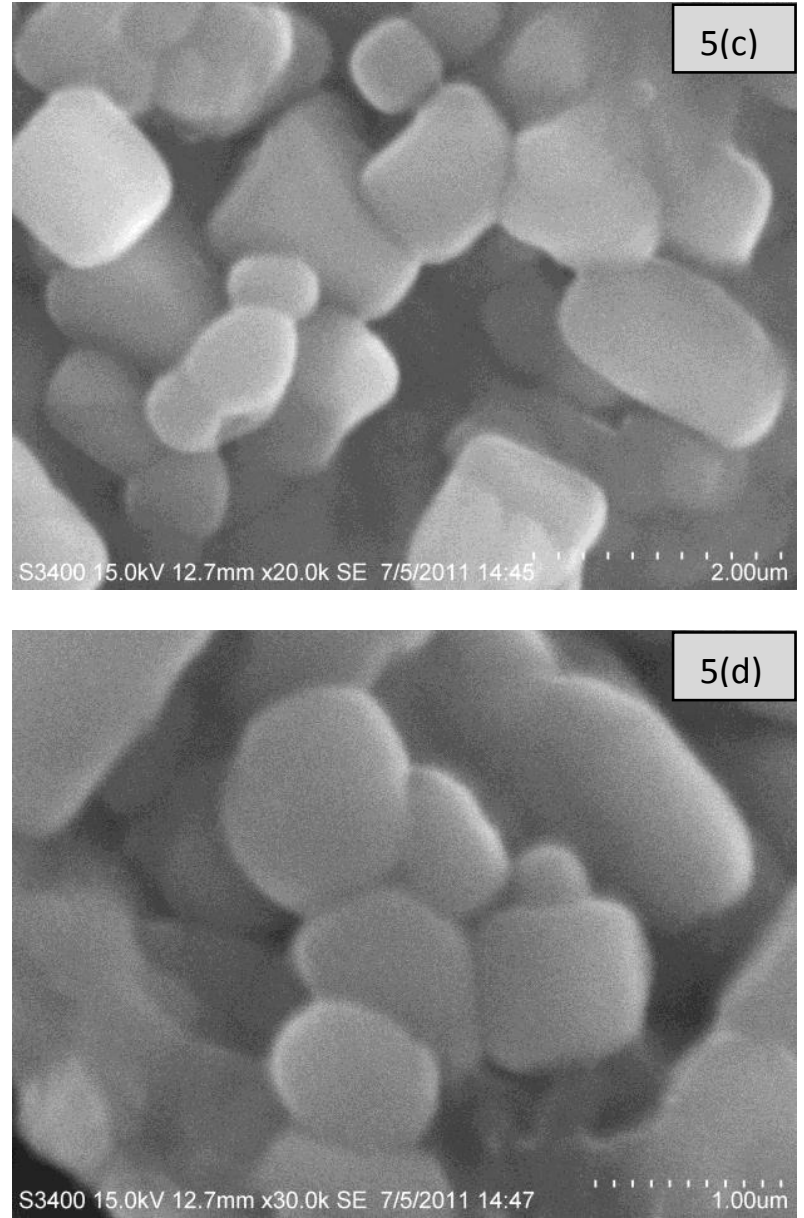

Fig.5. SEM images of TGPC without Urea doped

\section{CONCLUSION}

The Non Linear Optical Semiorganic urea doped, undoped Triglycine potassium chloride single crystal was grown by slow evaporation techniques. Its optical application was investigated by UV, FTIR, SEM studies. This investigation confirms that the crystal having good transparency when compared with urea doped crystal. Though it has slight variation in transparency, it can be used in NLO applications. Because there is no absorption peaks appears in its UV studies. This is the basic principle to study NLO properties. The functional groups were analyzed by its FT-IR Spectrums. It shows that, without urea doped titled crystal having no potassium chloride frequencies. Cause is that may be triglycine acting as a capping agent. But doping with urea glycine releases the $\mathrm{KCl}$ and absorbed Urea with in it. This result is having good agreement with and confirms by the investigation of SEM studies. And also adding Fertilizer in to titled crystal, its character gets some changes and also the grain size may be converted in to nano size.

\section{ACKNOWLEDGEMENT}

Author would like to thank Dr.A.Ravichandran, the Principal, Christ college of Engineering and Technology, Puducherry, for providing lab facilities and also additional bear and guidance. The author wish to thank Dr.Thilak Kumar, Professor of physics, Periyar Arts and Science, Cuddalore and 
Dr.A.Christy Ferdinand, Professor of physics, St.Joseph's college of Arts and Science, Cuddalore for the writing up of FTIR characterization. And finally would like to express his sincere thanks to A.S.Manikandan, T. Thirumazhisai and A. Martin Joseph, the professor of physics, Christ college of engineering and technology, Puducherry for giving more encouragement and done the first review in this research.
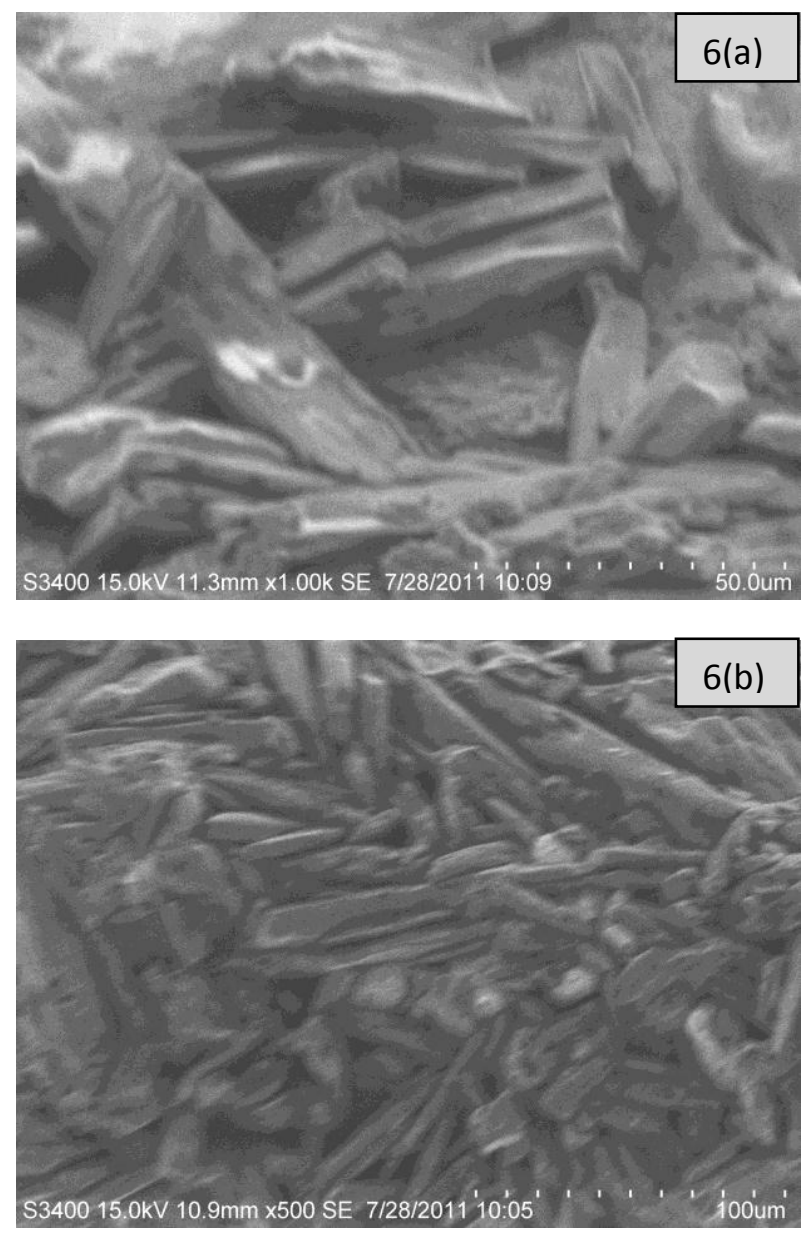

Fig.6. SEM images of TGPC doped with Urea

\section{REFERENCES}

[1]. S.Thiyagaraj, G.Meenakshi, Synthesis, Growth and UV Studies on Semi-Organic NLO Material by an impact of fertilizer (Tri-Glycine Potassium Chloride (TGPC) and Urea),Indian Streams Research Journal, 2, (2012), (II),14

[2]. M. N. Ravishankar, R. Chandramani, Optical and Mechanical characterization of Solution grown semiorganic NLO crystals Rasayan J.Chem. (2011) (1), 86-90

[3]. S. Gunasekaran, G. Anand, Crystal growth and comparison of vibrational and thermal properties of semiorganic nonlinear Optical materials. Pramana Journal of Physics, 75, (2010) (4), 683-690

[4]. M. Iyanar, C. Muthamizhchelvan, synthesis, growth and characterization of semiorganic nonlinear optical crystal: Glycine Zinc Chloride. Recent Research in Science and Technology 2, (2010) (1), 97-100

[5]. P.Kavitha, J. Shanthi, Optical Properties and Density Functional Theory Study of Single Crystal Glycine. International Journal of Applied Physics and Mathematics, 2, (2012), 4-9

[6]. A. J. Jeyaprakash Manoharan, N. Joseph John, Effect of amino acid doping on the dielectric properties of triglycine sulphate (TGS) crystals. Indian Journal of Science and Technology,

4, (2011), 6

[7]. G.Anbazhagan, P.S.Joseph, optical and electrical conductivity measurement of GLO single crystal for NLO application. Asian Journal of Information Technology, 11, (2012), (1), 36-39

[8]. Farhana Khanum and Jiban Podder, Growth, and Electrical Transport Properties of Pure and LiSO4Doped Triglycine Sulphate Crystal. International Journal of Optics, Synthesis, $\quad$ 20, (2012), 12-16

[9]. D.N.Sathiyanarayana, Vibrational Spectroscopy theory and Applications, New Age International Publishers. Ed. 1, 447, 2004

[10].D.N.Sathiyanarayana, vibrational spectroscopy theory and applications, New Age International Publishers. Ed.1, 448, 2004

[11]. D.N.Sathiyanarayana, Vibrational spectroscopy theory and applications, New Age International Publishers. Ed.1, 449, 2004 